\title{
Comparing the photophysics of the two forms of the Orange Carotenoid Protein using 2D electronic spectroscopy
}

\author{
E. De Re ${ }^{1,3}$, G.S. Schlau-Cohen ${ }^{2,3}$, V.M. Huxter ${ }^{2,3}$, R.L. Leverenz ${ }^{2}$, R.A. Mathies ${ }^{2}$ and G.R. \\ Fleming ${ }^{1,2,3}$ \\ ${ }^{1}$ Applied Science and Technology Graduate Group, University of California, Berkeley, 94720 \\ Berkeley, California USA \\ ${ }^{2}$ Department of Chemistry, University of California, Berkeley, 94720 Berkeley, California USA \\ ${ }^{3}$ Physical Bioscience Division, Lawrence Berkeley National Laboratories, 94720 Berkeley, \\ California USA
}

\begin{abstract}
Broadband two-dimensional electronic spectroscopy is applied to investigate the photophysics of the photoactive orange carotenoid protein, which is involved in nonphotochemical quenching in cyanobacteria. Differences in dynamics between the light and dark forms arise from the different structure of the carotenoid in the protein pocket, with consequences for the biological role of the two forms.
\end{abstract}

\section{Introduction}

Photosynthesis powers most life on earth by capturing solar energy and transferring the subsequent excitation to a location for conversion to chemical energy. If more light is absorbed than can be converted to chemical energy, oxygen species can be formed that endanger cellular components. Photosynthetic organisms employ mechanisms that safely dissipate excess energy as heat, collectively known as nonphotochemical quenching (NPQ). In cyanobacteria, the mechanisms of thermal energy dissipation remain elusive [1]; quenching has been shown to involve a photoactivated protein, the orange carotenoid protein (OCP) [2]. OCP is a water-soluble protein that non-covalently binds a single pigment, the carotenoid 3'-hydroxyechinenone. When blue-green light is absorbed, a conformational change in the pigment takes place, inducing a change in the absorption of the OCP from its inactive orange form $\left(\mathrm{OCP}^{\circ}\right)$, to a red active form $\left(\mathrm{OCP}^{r}\right)$. The red form has been shown to bind to the phycobilisome antenna and induce quenching of its fluorescence [3].

We use two-dimensional electronic spectroscopy to probe the absorption and relaxation pathways by excitation of the $S_{0} \rightarrow S_{2}$ transition of the carotenoid. These experiments investigate: 1) the photophysics of a carotenoid in a protein environment on ultrafast timescales (OCP is an ideal model system due to its single carotenoid); 2) the differences in excited state dynamics between the dark (orange) and light (red) form to gain insight into the interaction between the carotenoid and the surrounding protein pocket, with consequences for the photo-induced dissipation mechanism.

\section{Experimental Methods}

The apparatus used to collect the 2D spectra has been described in detail previously [4, 5]. Briefly, a

This is an Open Access article distributed under the terms of the Creative Commons Attribution License 2.0, which permits unrestricted use, distribution, and reproduction in any medium, provided the original work is properly cited. 
home-built Ti:sapphire regenerative amplifier laser system pumps a home-built non-collinear optical parametric amplifier, which produces laser pulses centered at $540 \mathrm{~nm}$ or $550 \mathrm{~nm}$ with $60 \mathrm{~nm}$ FWHM. The pulses are compressed to 12 fs via a prism compression line followed by a diffractionbased SLM pulse shaper. The laser beam is split into four pulses, focused on the sample in a box geometry. The interaction with pulses 1,2 and 3 generates a third-order signal in the phase-matched direction, collinear with beam 4, the local oscillator. This signal is spectrally dispersed and heterodyne-detected on a CCD camera. The delay between pulses 1 and 2 , the coherence time $\tau$, is scanned from -360 to $+360 \mathrm{fs}$ with a step of $0.8 \mathrm{fs}$, while the delay between pulses 2 and 3 , the waiting time $T$, is held fixed. The resulting array of interferograms collected for each value of $T$ is then Fourier-transformed and produces the final 2D spectrum, composed of the free induction decay (non-rephasing) and the photon echo (rephasing) signals. Dynamics are monitored by varying the waiting time $T$.

OCP was purified from a frozen paste of Arthrospira platensis cells [6]. The $\mathrm{OCP}^{\mathrm{r}}$ sample was prepared by mixing OCP (OD 30/cm) with chilled glycerol (30:70) under blue-green LED illumination. $\mathrm{OCP}^{\circ}$ was prepared in darkness. Repeated linear absorption measurements were performed to check for photoconversion. The samples were held in a $200 \mu \mathrm{m}$ quartz cell and cooled to $77 \mathrm{~K}$; the maximum OD was 0.28 at $502 \mathrm{~nm}$ for $\mathrm{OCP}^{\circ}$ sample, and 0.25 at $518 \mathrm{~nm}$ for $\mathrm{OCP}^{\mathrm{r}}$ sample.

\section{Results and Discussion}

Real valued, 2D relaxation spectra are shown for selected waiting times for $\mathrm{OCP}^{\circ}$ (Fig. 1(a)) and $\mathrm{OCP}^{\mathrm{r}}$ (Fig. 1(b)). The experimental results on the two forms suggest very different electronic structure, with distinct relaxation pathways and timescales.

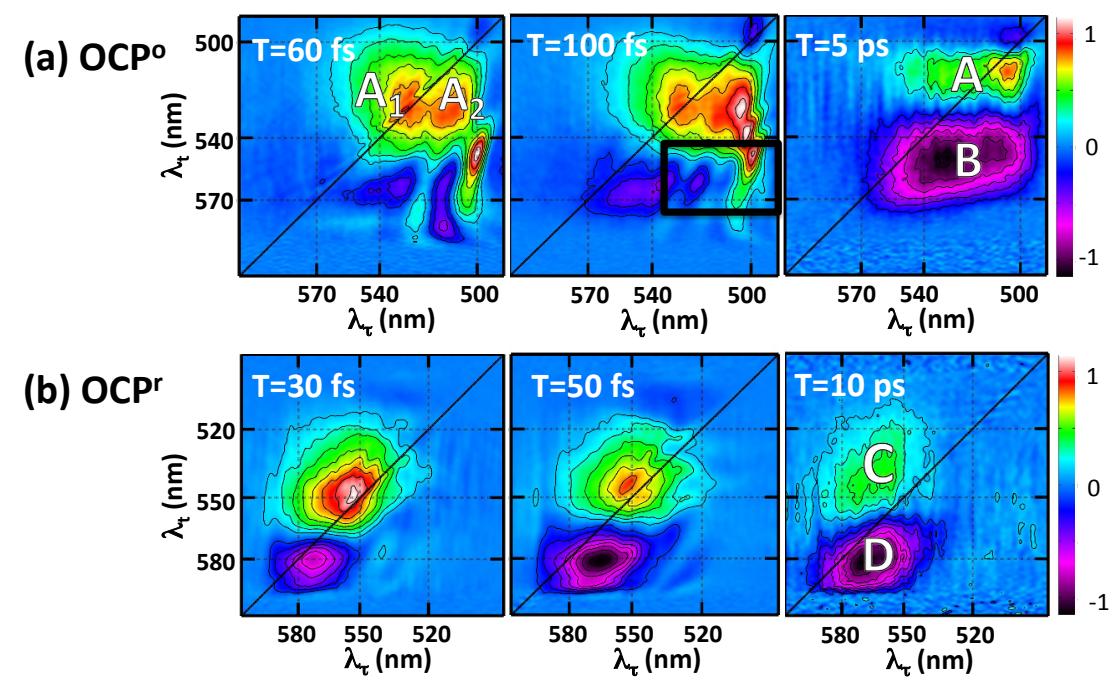

Fig. 1. 2D real relaxation spectra of $\mathrm{OCP}^{\circ}(\mathrm{a})$ and $\mathrm{OCPr}(\mathrm{b})$ at $77 \mathrm{~K}$ for selected waiting times $\mathrm{T}$.

Fig. 1(a) displays selected 2D spectra of $\mathrm{OCP}^{\circ}$. We excite the system into the $\mathrm{S}_{2}$ state, which appears as a positive feature centered around $515 \mathrm{~nm}$ (feature $\mathrm{A}$ in the $\mathrm{T}=5 \mathrm{ps}$ spectrum), corresponding to ground state bleach/stimulated emission signal from the $S_{0} \rightarrow S_{2}$ transition. Earlier time spectra are more structured, with multiple peaks contributing to the total signal. In the $\mathrm{T}=60$ and $\mathrm{T}=100 \mathrm{fs}$ spectra, we can distinguish the presence of two regions of separate intensity in the bleach region (features $A_{1}$ and $A_{2}$ in the $T=60$ fs spectrum). We assign their origin to the formation of ground state and excited state wavepackets, oscillating with a period corresponding to the separation 
in energy of the first vibronic feature of the carotenoid. The system then undergoes ultrafast internal conversion to the $S_{1}$ state, which appears as a negative feature at $\lambda_{t}=550 \mathrm{~nm}$ (feature $\mathrm{B}, \mathrm{T}=5 \mathrm{ps}$ ), corresponding to $S_{1} \rightarrow S_{N}$ excited state absorption. Additionally, in the early time spectra, we distinguish the presence of a region (boxed region in the $T=100 \mathrm{fs}$ spectrum) with multiple positive and negative peaks, overlapping spectrally and evolving in time. These oscillating features, lasting for $\sim 1 \mathrm{ps,} \mathrm{are} \mathrm{clearly} \mathrm{visible} \mathrm{in} \mathrm{the} \mathrm{rephasing} \mathrm{component} \mathrm{of} \mathrm{the} 2 \mathrm{D}$ spectra, while they are not visible in the non-rephasing portion of the spectrum. Given the spectral position and dynamics of these features being in accordance with previous experimental results obtained from $2 \mathrm{D}$ on $\beta$-carotene in solution, we hypothesize their assignment to the population of a hot ground state via an impulsive stimulated Raman scattering process [7]. The overall signal decays by 20 ps (data not shown), which we infer to be the timescale of relaxation from $S_{1}$ to $S_{0}$.

The dynamics in the active form, $\mathrm{OCP}^{\mathrm{r}}$, differ greatly from the dynamics just discussed in the inactive form, as seen by comparing Fig. 1(b) to Fig. 1(a). Excitation of the $S_{2}$ state is apparent at long times in the presence of a positive peak around $550 \mathrm{~nm}$, which we assign to bleach/stimulated emission from the $\mathrm{S}_{0} \rightarrow \mathrm{S}_{2}$ transition (feature $\mathrm{C}, \mathrm{T}=10 \mathrm{ps}$ ). The negative feature (feature $\mathrm{D}, \mathrm{T}=10 \mathrm{ps}$ ) corresponds to $S_{1} \rightarrow S_{N}$ excited state absorption. In contrast to the orange form, in the early time spectra we don't observe the presence of any vibronic features. From $T=30$ fs to $T=50$ fs we observe an increase of the intensity of the negative feature with respect to the positive feature, which we assign to population of the $S_{1}$ state.

\section{Conclusions}

We have presented results from broadband 2D electronic spectroscopy on the two forms of the photoactivated orange carotenoid protein, investigating the short-time dynamics of a carotenoid in a protein environment in a quenching and non-quenching conformation. We observed pronounced vibronic features and dynamics in the orange form, which we hypothesize are due to the carotenoid being held very tightly in the protein pocket. On the other hand, the red form spectra exhibit highly broadened peaks with no observable vibronic features. We hypothesize that the origin of this behaviour is due to the fact that, in $\mathrm{OCP}^{\mathrm{r}}$, the carotenoid is held very loosely in the protein pocket (a more open structure for the protein would facilitate the binding to the phycobilisome antenna) and has a continuum of vibrations which would increase the efficiency of energy dissipation.

\section{Acknowledgements}

This work was supported by the Director, Office of Science, and Office of Basic Energy Sciences of the U.S. Department of Energy under contract DE-AC02-05CH11231 and by the Division of Chemical Sciences, Geosciences, and Biosciences and Office of Basic Energy Sciences of the U.S. Department of Energy through grant DE-AC03-76SF000098.

\section{References}

1. K. El Bissati, E. Delphin, N. Murata, A.-L. Etienne, D. Kirilovsky, Biochim. Biophys. Acta 1457, 229-242 (2000).

2. D. Kirilovsky, Photosynth. Res. 93, 7-16 (2007).

3. A. Wilson, G. Ajlani, J.-M. Verbavatz, I. Vass, C.A. Kerfeld, D. Kirilovsky, The Plant Cell 18, 992-1007 (2006).

4. T. Brixner, I.V. Stopkin, G.R. Fleming, Opt. Lett. 29, 884-886 (2004).

5. G. S. Schlau-Cohen, T. R. Calhoun, N. S. Ginsberg, E. L. Read, M. Ballottari, R. Bassi, R. van

Grondelle, G. R. Fleming, J. Phys. Chem. B 113, 15352-15363 (2009)

6. T. Kay Holt, D.W. Krogmann, Biochim. Biophys. Acta 637, 408-414 (1981).

7. T.R. Calhoun, J.A. Davis, M.W. Graham, G.R. Fleming, Chem. Phys. Lett. 523, 1-5 (2012). 